\title{
Development of a Functional and Psychosocial Evaluation Toolkit Using Mixed Methodology in a Community-Based Physical Activity Program for Childhood Cancer Survivors
}

Jena Shank ( $\nabla$ jena.shank2@ucalgary.ca )

University of Calgary https://orcid.org/0000-0003-4353-2077

Carolina Chamorro-Viña

University of Calgary

Gregory Guilcher

Alberta Health Services

Fiona Schulte

Alberta Health Services

S. Nicole Culos-Reed

University of Calgary

\section{Research Article}

Keywords: Pediatric Oncology, Exercise, Community-Based Programs, Semi-Quantitative

Posted Date: July 19th, 2021

DOl: https://doi.org/10.21203/rs.3.rs-519550/v1

License: (c) (1) This work is licensed under a Creative Commons Attribution 4.0 International License.

Read Full License 


\section{Abstract \\ Purpose}

The evidence demonstrating the benefits of exercise and PA in patients and survivors of childhood cancer has been translated into a handful of community-based programs, such as the Pediatric cancer patients and survivors Engaging in Exercise for Recovery Program (PEER). In order to support the translation of research to practice, the next step in knowledge translation is to evaluate program effectiveness. An evaluation must consider the goals of the PEER program, feedback from key stakeholders and logistics of this program. Thus, the purpose of this study was to develop an evaluation toolkit with an algorithm for implementation for the PEER program.

\section{Methods}

Semi-structured interviews were conducted with three different groups (stakeholders in pediatric oncology, PEER parents and PEER participants). The interviews were transcribed and coded by two independent reviewers.

\section{Results}

Key themes extracted from the interviews were split into physical and psychosocial themes. The most commonly reported psychosocial themes were QOL, fatigue/energy levels, fun and confidence levels; and physical themes included motor skills, physical literacy and physical activity levels. Tools were compiled into the evaluation based on key themes identified as well as logistics of PEER. An algorithm was developed to tailor the evaluation to participants based on age, cognitive ability and mobility.

\section{Conclusion}

To date, this is the first evaluation toolkit and algorithm developed for a specific community-based PA program, the PEER program. The next step in knowledge-translation will be to implement the evaluation to assess feasibility, and share the evaluation for adoption within other developing programs.

\section{Introduction}

\section{Pediatric Oncology and Physical Activity}

Advances in treatments and early detection over the past 30 years have led to increased survival rates in children diagnosed with cancer [4]. However, therapy for childhood cancer is not without adverse effects. The potential acute and chronic complications of cancer and its treatment have been increasingly described, impacting physical and psychosocial health [8]. Increasing evidence supports the benefits of 
physical activity (PA) to aid in improving physical and psychosocial outcomes in pediatric oncology [5]. This supportive evidence has led to the development of a handful of community-based programs that move this research evidence to practice. One such program is the Pediatric cancer patients and survivors Engaging in Exercise for Recovery (PEER) Program [3].

\section{The PEER Program}

PEER is a community-based PA program for childhood cancer patients and survivors, aged 2-18 [3]. The main goal of the PEER program is to provide a safe and fun PA/exercise program for children and adolescents [3]. The program is committed to encouraging the adoption of PA, improving physical literacy, reduce the negative side effects of cancer and its treatments, improve QOL [3] and will favour the reinsertion of children in the community.

To promote the continued growth of the PEER program and increase its viability as a key supportive care service during the pediatric oncology journey, evaluation of the potential physical and psychosocial benefits of the program is essential. An evaluation aligned with the goals of the PEER program, may provide valuable feedback about the program's effectiveness and continue to be offered sustainably in the community. The PEER program evaluation of participant outcomes must include key stakeholders, including the health care professionals (HCP), families, program developers and administration operating the program [7]. Involving key stakeholders in development of the evaluation toolkit is critical to identify key themes such that they can be aligned with the goals of the program. Given the community-based nature of the program, it is important to balance the collection of psychometrically sound data, but also ensure the proposed evaluation is logistically feasible for the PEER program.

The purpose of this study was to (a) conduct interviews with HCPs, PEER families and users to gather feedback on the tools outlined in a previously described scoping review to see what stakeholders and users consider valuable and required components for evaluation [9] (b) based on the findings of the interviews and with consideration of the practicalities/goals of program delivery, to develop a toolbox and algorithm for implementation.

\section{Methods}

A scoping review compiled evaluation tools used to date in PA/exercise studies and programs in childhood cancer [9]. This review was used to guide interviews with key stakeholders to further understand the potential participant outcomes they want to see reported from the PEER program or value from PEER participation [9]. The results of both the review and the interviews guided the current work, the compilation of an evaluation toolkit and algorithm for implementation within the PEER program.

\section{Participants}

Interviewees were comprised of three categories. First, key stakeholders in pediatric oncology (HCPs) at the Alberta Children's Hospital and within the PEER program. Of note, two of the collaborators of the PEER 
Program were also HCPs, and thus were interviewed with consideration of both of these roles. Potential HCP interviewees were approached based on their involvement in the PEER program, in addition to using snowball recruitment to approach HCPs in pediatric oncology who were not aware of the PEER program. Individuals interested in participating were contacted via email to set up an interview, and those who were unable to meet in person were invited to set up a phone interview. Second, parents in the PEER program (all age groups) were emailed by the PEER program developer/coordinator (CCV), inviting them to participate in a 10-15 minute interview. Third, school-aged children and adolescents in the PEER program were invited to participate in an interview. Parents were asked to attend the interview with the children and adolescents. Participants were fully informed of the study requirements and provided written informed consent, and those under 18 years of age provided assent or parental consent. The Health Research Ethics Board of Alberta (HREBA) Cancer Committee approved this study (HREBA.CC-17-0094).

\section{Procedure}

\section{Interview}

Interviews lasted 10-30 minutes and were either at a location convenient to the participant or over the telephone. Interviews were conducted with each participant individually, except for children in the PEER program, in which case his/her parent was present. The interviewer (JAS) gave a description of the purpose of the study, provided a list to HCPs of potential evaluation tools from the scoping review [9] and provided time to answer any questions before the interview. The interviewer had a set of topics to guide the semi-structured interview. The interviews were customized to different age groups or key stakeholders in the program. For example, children were asked, "Why do you enjoy coming to the PEER program?" compared to parents who were asked, "What do you want your child to get out of the PEER program (i.e. specific areas you find they need improvement both physical and psychosocial aspects)?" The interviews were designed to understand why children and parents like the PEER program and what themes they value in the program. Interviews with HCPs were also designed to determine what aspects of the PEER program they value. Thus, HCPs were asked more specific questions, such as "What do you believe are important physical and psychosocial themes to capture in the PEER program (i.e. QOL, physical literacy, motor proficiency, well-being)?", and the results of a scoping review performed prior to this study determined evaluation tools which were suggested to help guide/prompt the discussion.

\section{Transcription}

The interviewers were recorded using a digital recorder and transcribed verbatim. The interviewer (JAS) made notes during the interview to document key points and relevant information. Key themes and any logistical problems that arose from interviews were discussed between the interviewer (JAS) and second reviewer (CCV). Any disagreements among themes were discussed and resolved by a third reviewer (NCR).

\section{Analysis Tool}


Consistent with the thematic analysis approach, the analysis was split into 6 separate phases [1]. The data coding and extraction was performed using NVivo by two reviewers (JAS) and (CCV). Phase 1 included familiarization with data where the independent reviewer (JAS) read and re-read the data and generated a list of key ideas. These key ideas were discussed with the second reviewer (CCV), which lead into phase 2 where initial codes emerged [1]. Following this, the codes were grouped into broader themes in phase 3 , then they were continually refined and developed until both reviewers (JAS) and (CCV) agreed on the codes and themes. In phase 4, some themes were collapsed or removed [1]. Phase 5 involved creating the thematic map where the final set of themes are defined, which lead to phase 6 , the algorithm development. This was determined from themes as well as from the scoping review conducted [9] to describe the subset of evaluation tools each child should complete based on age, physical and cognitive ability, and taking into consideration overall the logistics of the PEER program implementation.

\section{Evaluation toolkit and algorithm development}

The evaluation tools for the current work were selected based on three criteria (a) results from the scoping review that allow us to gather information about assessments previously used in pediatric oncology and exercise research [9] (b) the themes identified from the interviews, that allow us to understand what participants and stakeholders want to know from an evaluation and (c) feedback from the PEER program developer/coordinator that allow us to understand if assessments selected fit within the logistics of delivery for the PEER program (cost, required personnel, duration, and objectives). All tools were also assessed based on psychometric properties, goals of the PEER program (i.e. an evaluation for a diverse group of participants) and logistics of implementation for specific use in the PEER program (i.e. cost, length of time to complete, required personnel). The goal is to implement the evaluation at 2-3 weeks after starting the PEER program (whenever the participant joins PEER, given rolling-recruitment), and then again after 18-weeks and 36-weeks of participation. This allows for one evaluation at a minimum per semester (Fall, Winter, Spring). In addition, an algorithm was developed to ensure participants received a systematic evaluation that was tailored to their needs.

\section{Results}

\section{Participants}

A total of 18 participants were approached and interviewed (Refer to Table 1). The interviewees included two pediatric oncologists, one pediatric psychologist, one pediatric occupational therapist, one PEER pediatric oncology nurse, the PEER developer/coordinator, Kids Cancer Care Outreach Specialist, two preschool group PEER parents, four school group PEER parents, one adolescent group PEER parent, three school aged PEER participants and one adolescent PEER participant. 
Table 1

Participant Characteristics

\begin{tabular}{|ll|}
\hline Participant Groups & Number of Participants \\
\hline Health Care Professionals (HCP) & 4 (2 oncologists, 1 psychologist, 1 occupational therapist) \\
\hline PEER Administrator (Admin) & 3 (KCC Outreach specialist; PEER Co-ordinator; PEER Nurse) \\
\hline PEER Parents & 7 (2 pre-school group, 4 school group and 1 adolescent group) \\
\hline PEER Participants & 4 (3 school aged, 1 adolescent) \\
\hline
\end{tabular}

Interviews

Interviews resulted in physical and psychosocial themes, as outlined below.

\section{Physical Themes}

The main physical themes identified in the interviews are identified in Table 2 . The most commonly reported physical themes for outcomes to assess in the PEER program as identified by parents, participants/users and HCPs were motor skills, physical literacy and physical activity levels. These were identified in $50-61 \%$ of the interviews. One HCP did not agree with having a formal evaluation of participant outcomes in the PEER program and thus did not identify any themes. 
Table 2

Physical and Psychosocial Themes for PEER Evaluation

Main Physical Sub-Themes

Themes

Physical

Themes
Adaptive Functioning: is the ability to cope with daily environmental demands both individually and in social interactions [9]

Balance
Number and Type of Source who Identified

2 (HCP, 1

Parent)

2 (1 HCP, 1

Parent)

2 (2 HCPs)

Activities of Daily Living: is the capacity to perform activities of daily living (i.e.climbing stairs and getting dressed) [3]

Developmental Milestones: behaviors and abilities that are exhibited through infancy and early childhood development [10]

Flexibility

3 (2 HCPs, 1

Parent)

1 (HCP)

Motor Skills

10 (5 HCPs, 5

Parents)

Neuropathy

2 (1 HCP, 1

Parent)

New Activities

7 (4 Parents, 3

PEER

Participants)

Pain

2 (2 HCPs)

Physical Activity Levels

11 (4 HCPs, 3

Parents, 1 PEER

Participant, 3

PEER admin)

Physical Literacy: "movement vocabulary", and is a combination of confidence, competence, and motivation to engage in a variety of sports and activities in all environments (i.e. indoors, outdoors, in all seasons) [11]

Proprioception

Strength

No themes

Psychosocial

Themes
Communication

Confidence

Coping Mechanisms
1 (HCP)

5 (3 HCPs, 2

Parents)

1 (HCP)

9 (4 HCPs, 2

Parents, 3 PEER admin)

1 (HCP)

7 (4 HCPs, 3

Parents)

1 (Parent) 
Fatigue/Energy

9 (3 HCPs, 3 Parents, 3 PEER admin)

Fun

9 (2 HCPs, 5

Parents, 2 PEER participants)

\section{Mood}

1 (HCP)

Quality of Life

8 (4 HCPs, 1

Parent, 3 PEER admin)

Social Support

3 (2 HCPs, 1

Parent)

Other (parental measures)

2 (1 HCP, 1

Parent)

No themes

1 (HCP)

\section{Psychosocial Themes}

The main psychosocial themes reported in the interviews are provided in Table 2. The most common psychosocial themes for outcomes identified were QOL, fatigue/energy levels, fun and confidence levels. Less commonly reported were mood, communication, social support, coping mechanisms and parental themes. Most HCPs reported wanting to measure QOL and fatigue, compared to parents mostly reported wanting to measure if their child has fun and levels of confidence to engage in PA.

\section{PEER program participant's Evaluation Toolkit}

The results from the scoping review and the interviews were compiled to create an evaluation toolkit and algorithm to assess PEER program participants (Refer to Appendix A). The thematic results from the interviews confirmed that the most valued/important themes to capture were (a) physical literacy, (b) motor performance, (c) developmental milestones, (d) QOL, (e) confidence, (f) PA levels, (g) exploring new activities, (h) fatigue/energy and, (i) fun (Refer to Table 3). Strength was also mentioned as an outcome of interest by 3 HCPs and 2 parents, however it was not included in the evaluation due to logistical constraints. The PEER program runs once a week and the frequency might be not enough to promote a statistic change in strength variables. The main objective of the program is increase physical literacy, motor development and QOL. Specifically, as there is limited time within the PEER program to implement the evaluation (i.e. one 1-hour session), the goals of the PEER program were prioritized over assessment of strength themes. While pain was less commonly mentioned, it was included because 2 HCPs discussed the emerging research on pain in pediatric oncology and the recommended pain measurement 
tool assesses how pain interferes with other important themes (i.e. engaging in PA, having fun, and QOL) [11]. Given the relative ease with which this tool may be implemented, it was included in the evaluation toolkit. 
Table 3

Themes and Evaluation Tools Selected For PEER

\begin{tabular}{|c|c|c|c|c|c|c|}
\hline $\begin{array}{l}\text { Important } \\
\text { Themes to } \\
\text { Assess }\end{array}$ & Evaluation Tools & $\begin{array}{l}\text { Age } \\
\text { Group }\end{array}$ & Duration & $\begin{array}{l}\text { Type of } \\
\text { Measure } \\
\text { (subjective } \\
\text { or } \\
\text { objective) }\end{array}$ & Cost & $\begin{array}{l}\text { Who can } \\
\text { administer }\end{array}$ \\
\hline $\begin{array}{l}\text { Physical } \\
\text { Literacy }\end{array}$ & $\begin{array}{l}\text { PLAYFun and } \\
\text { PLAYParent [11] }\end{array}$ & $>7$ & $\begin{array}{l}\text { Not } \\
\text { Reported }\end{array}$ & $\begin{array}{l}\text { Subjective } \\
\text { (child) or } \\
\text { Objective } \\
\text { (parent- } \\
\text { proxy) }\end{array}$ & Free & $\begin{array}{l}\text { Exercise or } \\
\text { rehab trained } \\
\text { individual }\end{array}$ \\
\hline $\begin{array}{l}\text { Motor } \\
\text { Performance }\end{array}$ & $\begin{array}{l}\text { Bruininks- } \\
\text { Oseretsky Test of } \\
\text { Motor } \\
\text { Proficiency: Brief } \\
\text { Version }-2 \text { nd } \\
\text { edition [13] }\end{array}$ & $>4$ & $\begin{array}{l}20-30 \\
\text { minutes }\end{array}$ & Objective & $\begin{array}{l}\$ 284.55 \\
\text { CDN }\end{array}$ & $\begin{array}{l}\text { Exercise or } \\
\text { rehab trained } \\
\text { individual }\end{array}$ \\
\hline $\begin{array}{l}\text { Developmental } \\
\text { Milestones }\end{array}$ & $\begin{array}{l}\text { KidSense Gross } \\
\text { Motor } \\
\text { Development [14] }\end{array}$ & $2-6$ & $\begin{array}{l}\text { Not } \\
\text { Reported }\end{array}$ & Objective & Free & $\begin{array}{l}\text { No required } \\
\text { qualifications }\end{array}$ \\
\hline QOL & $\begin{array}{l}\text { PedsQL Generic } \\
\text { Core [15] }\end{array}$ & $2-18$ & $\begin{array}{l}<5 \\
\text { minutes }\end{array}$ & $\begin{array}{l}\text { Subjective } \\
\text { (child) or } \\
\text { Objective } \\
\text { (parent- } \\
\text { proxy) }\end{array}$ & Free & $\begin{array}{l}\text { No required } \\
\text { qualifications }\end{array}$ \\
\hline Confidence & $\begin{array}{l}\text { PLAYParent and } \\
\text { PLAYSelf [11] }\end{array}$ & $>7$ & $\begin{array}{l}<5 \\
\text { minutes }\end{array}$ & $\begin{array}{l}\text { Subjective } \\
\text { (child) or } \\
\text { Objective } \\
\text { (parent- } \\
\text { proxy) }\end{array}$ & Free & $\begin{array}{l}\text { No required } \\
\text { qualifications }\end{array}$ \\
\hline PA Levels & $\begin{array}{l}\text { PLAYParent and } \\
\text { PLAYSelf [11] }\end{array}$ & $>7$ & $\begin{array}{l}<5 \\
\text { minutes }\end{array}$ & $\begin{array}{l}\text { Subjective } \\
\text { (child) or } \\
\text { Objective } \\
\text { (parent- } \\
\text { proxy) }\end{array}$ & Free & $\begin{array}{l}\text { No required } \\
\text { qualifications }\end{array}$ \\
\hline $\begin{array}{l}\text { Exploring New } \\
\text { Activities }\end{array}$ & $\begin{array}{l}\text { PLAYParent and } \\
\text { PLAYSelf [11] }\end{array}$ & $>7$ & $\begin{array}{l}<5 \\
\text { minutes }\end{array}$ & $\begin{array}{l}\text { Subjective } \\
\text { (child) or } \\
\text { Objective } \\
\text { (parent- } \\
\text { proxy) }\end{array}$ & Free & $\begin{array}{l}\text { No required } \\
\text { qualifications }\end{array}$ \\
\hline Fatigue/Energy & $\begin{array}{l}\text { PedsQL } \\
\text { Multidimensional } \\
\text { Fatigue Scale [16] }\end{array}$ & $2-18$ & $\begin{array}{l}<5 \\
\text { minutes }\end{array}$ & $\begin{array}{l}\text { Subjective } \\
\text { (child) or } \\
\text { Objective } \\
\text { (parent- } \\
\text { proxy) }\end{array}$ & Free & $\begin{array}{l}\text { No required } \\
\text { qualifications }\end{array}$ \\
\hline
\end{tabular}

Note: No required qualifications to administer the test, thus the assessment tool can be used for program evaluation and should not be used as a diagnostic tool. 


\begin{tabular}{|lllllll|}
\hline $\begin{array}{l}\text { Important } \\
\text { Themes to } \\
\text { Assess }\end{array}$ & Evaluation Tools & $\begin{array}{l}\text { Age } \\
\text { Group }\end{array}$ & Duration & $\begin{array}{l}\text { Type of } \\
\text { Measure } \\
\text { (subjective } \\
\text { or } \\
\text { objective) }\end{array}$ & Cost & $\begin{array}{l}\text { Who can } \\
\text { administer }\end{array}$ \\
\hline Fun & $\begin{array}{l}\text { PLAYParent and } \\
\text { PLAYSelf [1] }\end{array}$ & $>7$ & $\begin{array}{l}<5 \\
\text { minutes }\end{array}$ & $\begin{array}{l}\text { Subjective } \\
\text { (child) or } \\
\text { Objective } \\
\text { (parent- } \\
\text { proxy) }\end{array}$ & Free & $\begin{array}{l}\text { No required } \\
\text { qualifications }\end{array}$ \\
\hline Pain & $\begin{array}{l}\text { PROMIS Pain } \\
\text { Scale [17] }\end{array}$ & $>5$ & $\begin{array}{l}<5 \\
\text { minutes }\end{array}$ & $\begin{array}{l}\text { Subjective } \\
\text { (child) or } \\
\text { Objective } \\
\text { (parent- } \\
\text { proxy) }\end{array}$ & Free & $\begin{array}{l}\text { No required } \\
\text { qualifications }\end{array}$ \\
\hline $\begin{array}{l}\text { Note: No required qualifications to administer the test, thus the assessment tool can be used for } \\
\text { program evaluation and should not be used as a diagnostic tool. }\end{array}$ & & \\
\hline
\end{tabular}

\section{Algorithm}

The evaluation tools were organized into an algorithm for implementation, considering the needs of the participants in the PEER program (i.e. age, cognitive ability and mobility) and within the logistics of the PEER program (i.e. duration). The evaluation was split into an online portion and an in-session assessment. This makes the assessment of the desired themes feasible within the 1-hour time constraints of the PEER program session (Refer to Appendix A). Specifically, all questionnaires were included in the online portion and all physical assessments were included in the in-session portion. The evaluation tools were split into 4 age groups, given the assessment tools were created for the following age groups, (a) 2-4, (b) 5-7, (c) 8-12, and (d) 13-18. There are approximately 36 PEER program sessions a year (September-June). For participants aged 2-12, a parent proxy report would be completed online, while older participants may complete their own. Given the diversity of PEER, it is important to ensure all participants receive an evaluation tailored to their ability. Thus, the participants were further split based on both cognitive ability and mobility (see Appendix A for the tailoring options).

Specifically, based on feedback from a pediatric psychologist, the level of cognitive ability was defined as a participant who receives full-time special services in the classroom. For mobility, a cancer and exercise specialist, occupational therapist and physiotherapist were consulted. Neither the literature to date nor pediatric exercise specialists knew how to quickly assess mobility without a formal evaluation. Thus, the PEER program coordinator, who is a cancer exercise specialist and has a $\mathrm{PhD}$ in biomedicine and health, and a kinesiologist, defined upper and lower body mobility restrictions and mobility assistance (Refer to Appendix A). These definitions were based on ensuring the validity of the assessment tools were not compromised (i.e. a child could complete a throwing task, but requires assistance to stand, thus the upper body assessment would not be compromised by lower body assistance). 
The evaluation toolkit includes psychosocial tools to assess QOL and fatigue (using the PedsQL Generic Core and Multidimensional Fatigue Scale, participant or parent-proxy measures) for all age groups and pain (using the PROMIS) starting at age 7 and above (as it is not available for younger ages). In addition, the toolkit provides an assessment for confidence to engage in PA and participation in a variety of activities, for children aged 7 and up using the PLAYSelf and PLAYParent. For children with severe cognitive deficits, a session observation assessment was determined to be a more valid and thus appropriate assessment tool for motor skills and social interaction in the PEER program (Refer to Appendix B). The session observation assessment allows the evaluator to set goals for the child to complete in the session, specific to his/her needs.

For the motor development assessment and or physical literacy, participants aged 2-6 years complete a developmental milestones assessment to see if they are reaching the sufficient gross motor milestones for their age, which also includes aspects of physical literacy. To date, there is no evaluation tool that assesses physical literacy below the age of 7 , and thus the gross motor developmental milestones were utilized. Participants aged 7-18 with no mobility or cognitive restrictions complete a physical literacy assessment, the PLAYFun tools. Physical literacy was a commonly discussed outcome amongst interviewees, however there were no assessment tools used to date that measured physical literacy in the childhood cancer and exercise programs or studies [9]. However, a scoping review reported on evaluation tools used assess physical literacy in all children, youth and adult populations [6]. It was reported that when defining physical literacy it may be beneficial to use a more holistic and integrated definition [6]. For the purpose of this work, none of the evaluation tools gathered in the scoping review fit within the logistics of the PEER program (i.e. time constraints, inappropriate age range, themes do not align with the PEER program goals) [6]. Therefore, the Physical Literacy Assessment for Youth Tools (PLAYTools), developed by the Sport for Life Society, were selected to assess physical literacy [10]. It is important to note that the PLAYTools has not yet been reported in the cancer and exercise literature [10]. Finally, for children aged 4-18 that display mobility limitations, the Bruininks-Oseretsky Test of Motor Proficiency (BOT 2) was recommended to provide a more validated and extensive evaluation of motor performance [2].

\section{Discussion}

Early research has established the benefits of PA and exercise in pediatric oncology [5]. However, this has led to only limited translation of research to community-based PA programs, such as the PEER program [4]. Earlier work on the PEER program evaluation has determined its feasibility and safety [3]. In order to assess the effectiveness of the PEER program, an evaluation of the program's potential benefits is necessary. Given the community-based nature of PEER, consideration of the logistics and participant characteristics (age, cancer type, cancer treatment, cognitive ability and mobility) must be taken into account. The results of the interviews, along with the scoping review, guided the current work, the development of an evaluation toolkit and algorithm for implementation within the PEER program [9]. The final evaluation toolkit and algorithm (Appendix A) have important research and clinical implications. 
First, from a research perspective, development and implementation of an evaluation toolkit can aid in understanding the effectiveness of community-based PA/exercise programming in childhood cancer. The toolkit provides a "starting point" to assess the PEER participants and corroborate the effectiveness of community-based programs, such as the PEER program. This allows for the necessary adaptations to the specific goals and logistics within a specific program to be considered. Additionally, while most evaluation tools selected have been widely used and accepted in childhood cancer, some evaluation tools have yet to be reported in the literature (healthy or cancer specific populations). For example, the PLAYTools have been developed by a panel of health and exercise experts, but have yet to be reported in the literature. Thus, continued application of the PLAYTools in the PEER program, may provide feedback regarding the validity of and reliability of the tools.

From a clinical perspective, the evaluation toolkit can provide objective assessment of key patient themes that can be provided to both the health care team and the families. Given the nature of community-based programs such as PEER, this clinical feedback is both valuable and extremely important in terms of both patient themes and maintaining the ongoing value of the program. Ongoing evaluation within the PEER program specifically ensures that each participant receives an assessment tailored to their needs and that will assess potential benefits from participation. Participants, parents and their HCPs will receive feedback from the evaluation, allowing tracking of progress/improvements over time. In addition, regularly seeing the impact of the PEER program participation on their patients may facilitate referral from HCPs into the program.

\section{Limitations}

There are a few important limitations to note regarding the evaluation tools selected. First, the evaluation toolkit and algorithm were specially designed for the PEER program and may not be applicable to all community-based PA/exercise programs for childhood cancer. For example, the evaluation tools were selected in alignment with available resources at the PEER program and the goals of the program. Implementation of the toolkit to any specific program must take into account that program's unique needs. Second, given the limited number of interviews conducted, the results may not clearly represent the views of all HCPs and PEER program participants/families. Third, the algorithm does not account for individual limitations such as osteoporosis or peripheral neuropathy, thus a trained professional is required to use discretion to ensure any individual alterations are made. The evaluation tool should be applied in a manner that provides valuable feedback to the participant/family and provides substantive evidence to the HCP with regards to the potential role of PA/exercise in pediatric oncology. Lastly, the evaluation tools were selected based on a number of criteria (psychometric properties, cost, duration and personnel required) and thus there is a challenge in selecting tools that meet all criteria. For example, the PLAYTools best suited the needs of the PEER program, but have yet to be reported in childhood cancer. While we deemed the PLAYTools suitable for use, future research on physical literacy in childhood cancer may provide a more suitable measure.

\section{Implications of Research Findings}


Despite these limitations, there are also important strengths to be noted. To date, there is nothing in the literature specifically evaluating community-based PA/exercise programs in pediatric oncology. The generated toolkit and algorithm may provide a framework for programs to utilize, thus enhancing our knowledge about the effectiveness of such programs. Lastly, consistent application of the toolkit may provide further feedback regarding the reliability and validity of the evaluation tools.

\section{Declarations}

\section{Funding}

This research study was funded by the Canadian Institute of Health Research (CIHR) and Alberta Children's Hospital Research Institute (ACHRI).

\section{Conflicts of Interest}

The authors have no conflict of interest to declare.

\section{Availability of Data and Material}

All identifying information was removed from data before manuscript preparation. Participants who were interested in study results will be provided with the final manuscript.

\section{Code Availability}

Not applicable.

\section{Authors' Contributions}

Ms. Shank conducted the research project for her MSc thesis and wrote the manuscript. Dr. ChamorroViña assisted with data collection and provided feedback and guidance throughout. Dr. Guilcher and Dr. Schulte were mentors and provided feedback on Ms. Shank's thesis. Dr. Culos-Reed was Ms. Shank's MSc supervisor and provided feedback and mentorship throughout her graduate work.

\section{Ethics Approval}

This research study was approved through the University of Calgary Research Ethics Board (HREBA.CC17-0094).

\section{Consent to Participate}

All participants signed an informed consent or assent form. All participants were informed, they may withdraw from participation at any point.

\section{Consent for Publication}


All participants were informed about the research project and that data would be published, which was outlined in the informed consent form. If participants were interested in published results, this would be provided to them upon publication.

\section{References}

1. Braun V, Clarke V (2006) Using thematic analysis in psychology Qualitative research in psychology 3: 77-101

2. Bruininks RH (2005) Bruininks-Oseretsky Test of Motor Proficiency, (BOT-2) Minneapolis. Pearson Assessment, MN

3. Chamorro Viña C, Guilcher GMT, Schulte F, De Vries A, Schwanke J, Culos-Reed SN (2017) Description of a Community-Based Exercise Program for Children With Cancer: A Sustainable, Safe, and Feasible Model Rehabilitation Oncology 35

4. Chamorro Viña C, Wurz AJ, Culos-Reed SN (2013) Promoting physical activity in pediatric oncology. Where do we go from here? Frontiers in oncology 3:173

5. Chamorro-Viña C, Keats M, Culos-Reed SN (2015) Pediatric Oncology Exercise Manual (POEM) Professional Version, 1st edn. Health \& Wellness Lab, Calgary

6. Edwards LC, Bryant AS, Keegan RJ, Morgan K, Cooper S-M, Jones AM (2018) 'Measuring' Physical Literacy and Related Constructs: A Systematic. Review of Empirical Findings Sports Medicine 48:659-682

7. Kronlund LJ (2018) Addressing Key Stakeholders to Assess the Reach of a Community-Based Pediatric Oncology Physical Activity Program. Graduate Studies, University of Calgary

8. Oeffinger KC, Mertens AC, Sklar CA, Kawashima T, Hudson MM, Meadows AT, Friedman DL, Marina N, Hobbie W, Kadan-Lottick NS, Schwartz CL, Leisenring W, Robison LL, Childhood Cancer Survivor S (2006) Chronic Health Conditions in Adult Survivors of Childhood Cancer The New England Journal of Medicine 355: 1572-1582

9. Shank J, Chamorro-Viña C, Guilcher GMT, Langelier DM, Schulte F, Culos-Reed SN (2019) Evaluation Tools for Physical Activity Programs for Childhood Cancer Survivors. A Scoping Review Journal of Pediatric Oncology Nursing

10. Sport for Life Society (2018) Physical Literacy Assessment for Youth. Physical Literacy. https://physicalliteracy.ca/portfolio-view/physical-literacy-canada-tools-assessment/. Accessed 15 April 2018

11. Varni JW, Stucky BD, Thissen D, DeWitt EM, Irwin DE, Lai J-S, Yeatts K, DeWalt DA (2010) PROMIS Pediatric Pain Interference Scale: An Item Response Theory Analysis of the Pediatric Pain Item Bank. J Pain 11:1109-1119

\section{Supplementary Files}


This is a list of supplementary files associated with this preprint. Click to download.

- InterviewsAppendix.docx 